
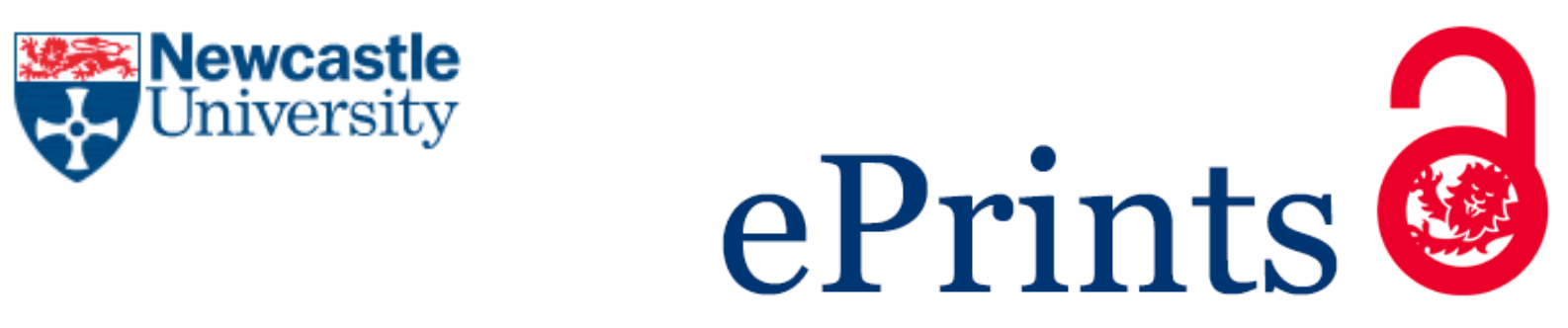

Xu YM, Jia YJ, Ai MM, Wang YD.

Heat transfer characteristics of external ventilated path in compact high-

voltage motor.

International Journal of Heat and Mass Transfer 2018, 124, 1136-1146

Copyright:

(C) 2018. This manuscript version is made available under the CC-BY-NC-ND 4.0 license

DOI link to article:

https://doi.org/10.1016/j.ijheatmasstransfer.2018.04.034

Date deposited:

$25 / 04 / 2018$

Embargo release date:

08 April 2019

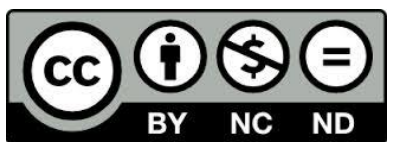

This work is licensed under a

Creative Commons Attribution-NonCommercial-NoDerivatives 4.0 International licence 


\title{
Heat Transfer Characteristics of External Ventilated Path in Compact High-voltage Motor
}

\author{
Yongming XU ${ }^{1 *}$, Yajie JIA ${ }^{1}$, Mengmeng $\mathrm{Ai}^{1}$, Yaodong Wang ${ }^{2}$ \\ (1.School of Electrical \& Electronic Engineering, Harbin University of Science and Technology, \\ Harbin 150080, Heilongjiang Province, China; \\ 2. School of Engineering, Newcastle University, Newcastle upon Tyne, NE1 7RU, UK) \\ Corresponding author. Tel:+86 13945696426. \\ E-mail address: xuyongming@ @rbust.edu.cn.
}

\begin{abstract}
This paper investigates the heat transfer characteristics of the external ventilated path of a compact, high-voltage, 4-pole, $2500 \mathrm{~kW}$ motor using flow-thermal coordination mechanism. A computational model is set up and validated by experimental test results. A series of simulation is performed. It is found that the deflection angle $\alpha$ and outlet angle $\beta$ of the fan blades are the key parameters affecting the efficiency of the cooling effect of the fan. Optimal measures are adopted by changing the deflection angle and outlet angle of fan blades. External fan efficiency is improved from $28.80 \%$ to $29.96 \%$ and outlet flow is increased by 0.08 $\mathrm{m}^{3} / \mathrm{s}$ by optimizing the deflection angle $\alpha$ and outlet angle $\beta$. It is found that the temperature of hyperthermal fluid of inner ventilated path is decreased from $75^{\circ} \mathrm{C}$ to $53.7^{\circ} \mathrm{C}$; at the same time the temperature of cryogenic fluid of external ventilated path is increased from $23^{\circ} \mathrm{C}$ to $49.4^{\circ} \mathrm{C}$. The outlet temperature of internal fluid of post-optimized cooler is dropped by $3^{\circ} \mathrm{C}$, and the external fluid temperature is increased by $2.5^{\circ} \mathrm{C}$. The performance of fan and the cooling effect is improved. The results from this study can provide an effective method for structural optimal design of compact high-voltage motors.
\end{abstract}

Keywords: Compact high-voltage motor, External ventilated path, Cooler, Heat transfer characteristic, Optimal design 


\section{Introduction}

With the development of the technologies, design of electric motors with high efficiency and high power density become popular. A compact high-voltage motor has the advantages of compact structure, high power density and high electromagnetic load. However, the increase of power density is bound to cause more serious overheating problem, which will reduce the motor efficiency. As a solution, controlling the range of temperature rise is a key factor affecting the efficiency of the overall design ${ }^{[1]}$. Therefore, it is necessary to analyze the fluid field and temperature field accurately and improve the cooling system capacity to ensure reliable operation of motor. The ventilated structure of a compact high-voltage motor is divided into two: internal and external ventilated path. The external ventilated path mainly takes away the excess heat inside the motor and become the key area to research fluid flow and heat transfer characteristic ${ }^{[2]}$. At present, many researchers focus their research interests on heat transfer and cooling of motor. Chang et al ${ }^{[3]}$ studied the thermal characteristics of enclosed air-to-air cooled motor using experimental and numerical simulation methods and found that the optimal design of cooling fan may improve the operation reliability of the motor. $\mathrm{Li}^{[4]}$ studied a permanent magnet electric motor with a centrifugal impeller and he found that the cooling air flow was proportional to the speed of the motor; and the rotational speed is inversely proportional to the external load; the heat transfer of armature surface indicated that the radial temperature change was slight but the axial change was noticeable.

The effect of cooling fan were studied in detail by a number of researchers [5-7]. It was found that the blade thickness of the fan had great influence on the performance and cooling effect of ventilated structure. Modelling three-dimensional (3D) temperature field of Totally Enclosed Fan Cooled (TEFC) motor were used to analyze the influence of rotor-bar damage by motor temperature [8-10] and it was found that the higher the temperature was, the larger the thermal stress was, and the uneven heat stress directly led to broken bar fault and influenced the motor life.

A 3-D coupled-field finite-element method for prediction of the temperature rise in air-cooled induction motors, considering the effect of rotor rotation on air convection, was proposed and validated by experimental tests by Zhang et al ${ }^{[11]}$. The multi-component fluid model can solve the problem that the velocity on interface between fluid and solid. A combined 2D-3D finite-element method was used to analyze temperature rise of high-speed permanent-magnet machine ${ }^{[12]}$. The thermal convection coefficient and air temperature rise was estimated by $2 \mathrm{D}$ multiphasic method and the temperature rise distribution in whole solid domain of the machine was determined by the 3D numerical heat-transfer method; and it was found that the method can get an accurate estimation of the local temperature rises. Kim et al ${ }^{[13]}$ investigated the influence of air-gap flow heating on the thermal characteristics of stator and windings in large-capacity induction motors using numerical simulation. Air-gap flow heating phenomena were defined and classified into three states: under-heating, over-heating, and super-heating and further research was carried out to identify the influence of the over-heating and super-heating states of the air-gap flow 
on the stator and windings. A thermal model of direct cooling motor was established by Nategh, S., et al ${ }^{[14]}$ using the lumped parameter and limited computational fluid dynamics (CFD) method. Experimental evaluation showed that the temperature distribution and hot-spot temperatures in the end winding under different loss levels and coolant flow velocity can be predicted accurately by the proposed lumped parameter thermal model. Torriano, F., et al ${ }^{[15]}$ studied the effect of rotation on heat transfer mechanisms in rotating machines using a hybrid numerical-experimental approach. The results from their study showed that an asymmetric profile in the tangential direction closer to the trailing edge due to the presence of a flow recirculation zone and the heat transfer profiles indicated that the highest values of heat transfer coefficient were in an intermediate region. The results from their study also showed that the heat transfer coefficients along the pole face at $300 \mathrm{rpm}$ average about four times those at $50 \mathrm{rpm}$. A coupled analytical and numerical method was presented to study thermal characteristics of a $37 \mathrm{~kW}$ induction motor by Nair, D. et al [16]. Their thermal network and 3D numerical model can be used to predict the accurate temperature distribution of the motor.

Although there were fruitful research results on fluid flow and heat transfer of electrical machine, there are few studies are found on fluid flow and heat transfer of the external ventilated path in high-voltage motors. In this research, the fluid flow and heat transfer characteristics of the external ventilated path for a compact, medium high-voltage, 4-pole, $2500 \mathrm{~kW}$ motor is modelled and analyzed based on the flow-thermal synergistic mechanism. An optimal external ventilated path and the simulation is presented. In addition, the accuracy and rationality of the modelling simulation results are verified by the experiment.

\section{Methodology}

The external ventilated path of a compact high-voltage motor includes an external fan and a cooler. The cooler is a place in which the heat exchange will happen between the hot air of internal ventilated path and the cold air of external ventilated path. The temperature of cryogenic fluid is increased while that of hyperthermal fluid is decreased. The ventilated system of compact high-voltage motor is shown in Fig.1.

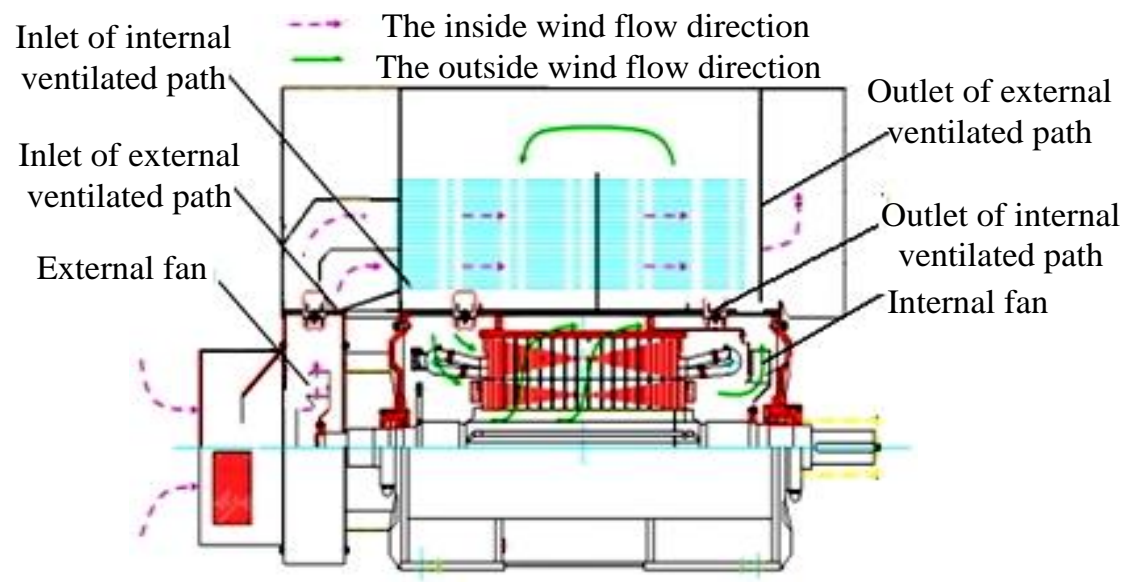


Fig.1 ventilated path structure of compact high voltage motor

In Fig.1, the green arrows represent the flow direction of internal ventilated path fluid, whereas the pink and dotted arrows represent the flow direction of the external ventilated path fluid. The motor ventilated path is a closed structure. Under the action of the internal fan, the internal high temperature fluid (green arrows) flows, and under the action of the external fan, the low temperature fluid (pink arrows) flows. The heat transfer between internal and external fluid takes place in the cooler ${ }^{[17]}$. The compact high-voltage motor prototype is shown in Fig.2.

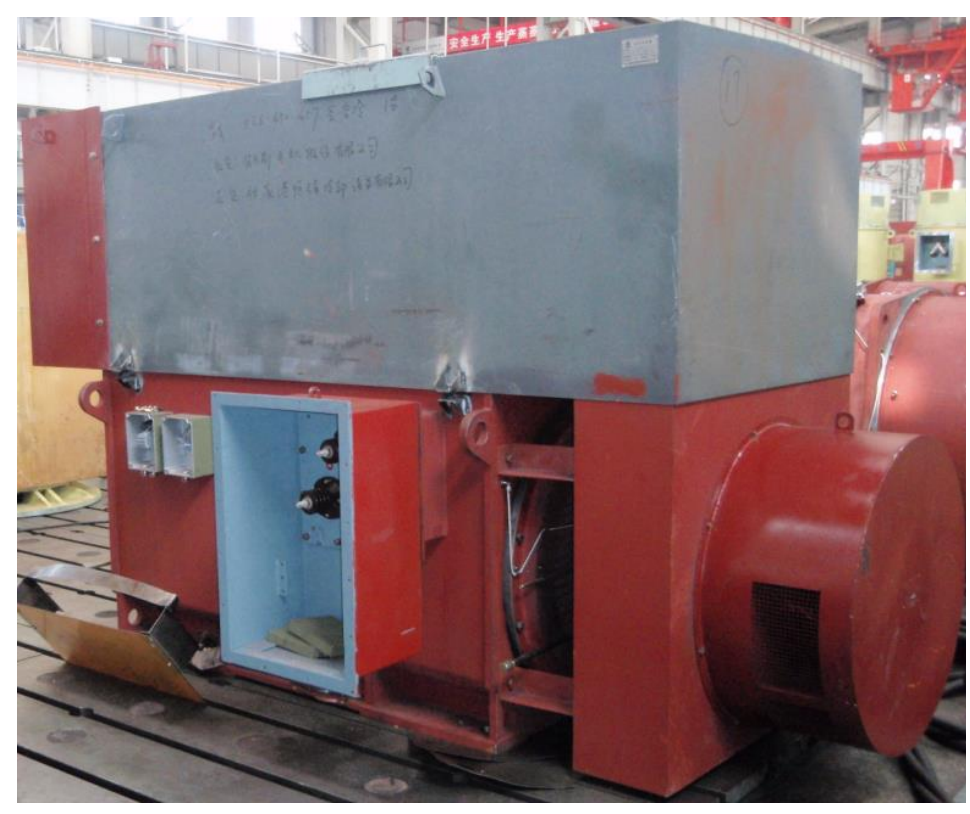

Fig.2 Compact high-voltage motor prototype

\subsection{Mathematic model of the external ventilated path}

The fluid flow is governed by the laws of conservation of physics, including the law of mass conservation, the law of conservation of momentum and the law of conservation of energy (Computational fluid dynamics analysis). The equation can be found in [19].

Meanwhile, the fluid flow has turbulence in it. Therefore it is necessary to introduce the turbulence equation ${ }^{[20]}$. The rotation effect in the mean flow is considered in Renormalization Group (RNG) $k-\varepsilon$ model which can make the calculated results more accurate ${ }^{[21]}$. So we choose it as the solution model. The corresponding $\mathrm{k}$ and $\varepsilon$ transport equations are:

$$
\begin{gathered}
\frac{\partial(\rho k)}{\partial t}+\frac{\partial\left(\rho k u_{i}\right)}{\partial x_{i}}=\frac{\partial}{\partial x_{j}}\left[\left(\alpha_{k} \mu_{e f f}\right) \frac{\partial k}{\partial x_{j}}\right]+G_{k}+G_{b}-\rho \varepsilon-Y_{M} \\
\frac{\partial(\rho \varepsilon)}{\partial t}+\frac{\partial\left(\rho \varepsilon u_{i}\right)}{\partial x_{i}}=\frac{\partial}{\partial x_{j}}\left[\left(\alpha_{\varepsilon} \mu_{e f f}\right) \frac{\partial \varepsilon}{\partial x_{j}}\right]+C_{1 \varepsilon} \frac{\varepsilon}{k}\left(G_{k}+C_{3 \varepsilon} G_{b}\right)-C_{2 \varepsilon} \rho \frac{\varepsilon^{2}}{k}-R
\end{gathered}
$$




$$
\left\{\begin{array}{l}
\mu_{e f f}=\mu+\mu_{t} \\
\mu_{t}=\rho C_{\mu} \frac{k^{2}}{\varepsilon}
\end{array}\right.
$$

Where $k$ is the turbulent kinetic energy, $\varepsilon$ is the dissipation rate of turbulent kinetic energy, $G_{k}$ is turbulent energy produced by mean velocity gradient, $\mu_{e f f}$ is the equivalent viscosity coefficient.

\subsection{Physical model of the external ventilated path}

The external fan of compact high-voltage motor is designed as rear-mounted. It mainly includes the front plate, the blade and the back plate with nine blades. Fig. 3 is the structure diagram of the external fan.

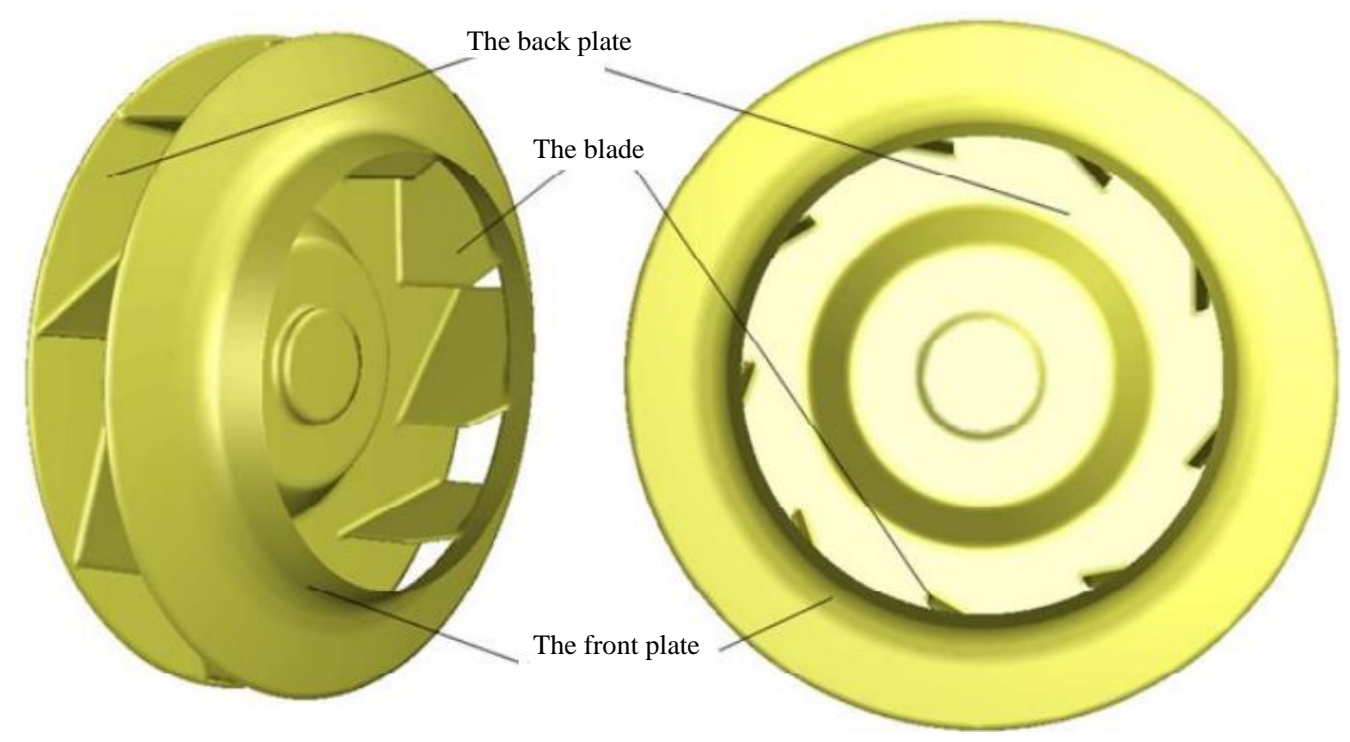

Fig.3. Structure diagram of the external fan

However, there is actually a great change in the cross-sectional area between the inlet and outlet of the fluid field of the external fan. Therefore, in order to make the fluid flow more realistic, it is necessary to establish a certain length of inlet and outlet without changing their area in fluid field mode ${ }^{[18]}$. A 3D solid model of external fan can be seen in Fig.4. 


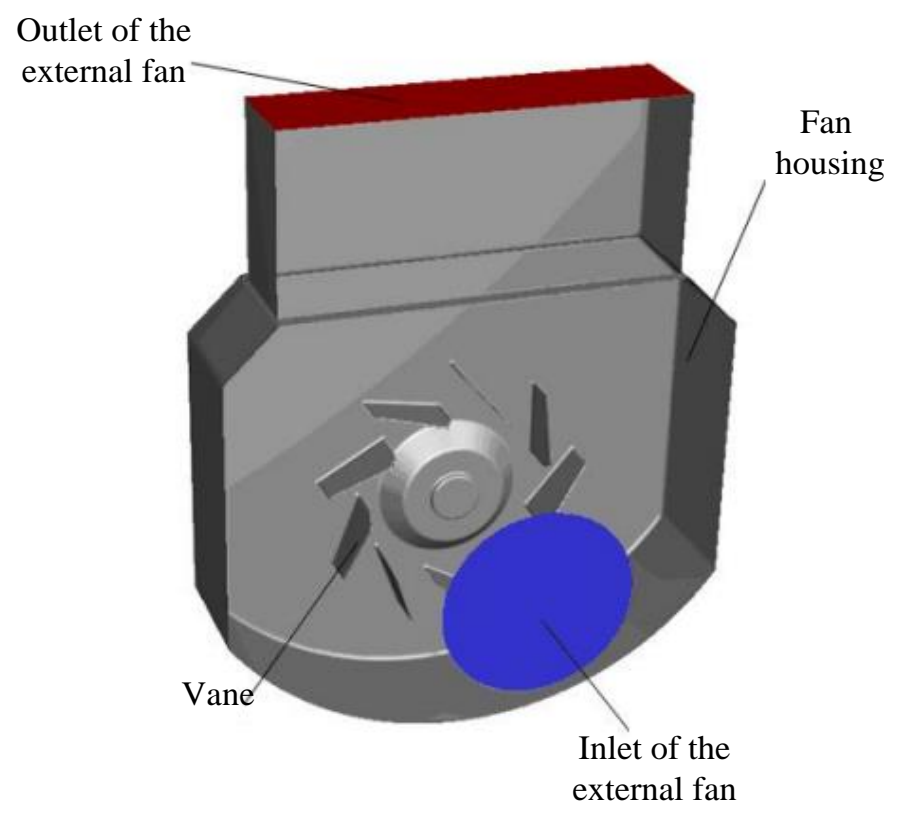

Fig.4. 3D solid model of external fan

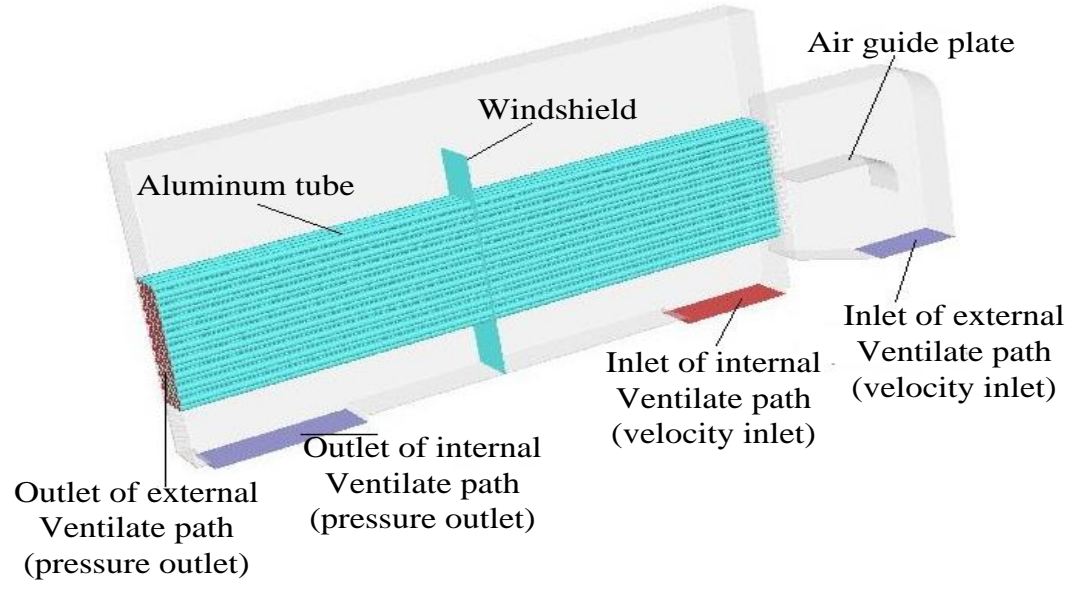

Fig.5. 3D model of cooler

The entire cooler used in this study has 750 cooling pipes and each pipe has a diameter of $20 \mathrm{~mm}$. The structure of the cooler is symmetrical. Therefore, a one-tenth model is chosen as the solver region to simplify the calculation. The $3 \mathrm{D}$ model of cooler is shown in Fig. 5.

\subsection{Basic assumptions and boundary conditions}

\subsubsection{Basic assumptions}

In order to run the 3D model, the following assumptions are used:

1) In the calculation area, the turbulent model is chosen to solve the calculation because of its large Reynolds number ${ }^{[22]}$.

2) The fluid flow velocity in the calculation area is much smaller than that of the sound speed. So it can be treated as an incompressible fluid ${ }^{[23] .}$ 
3) Assuming that all the cooling air outside the external fan is blown into the cooler $^{[24]}$.

\subsubsection{Boundary and initial conditions}

The boundary conditions are set as follows:

1) The inlet velocity is chosen as the inlet boundary condition of the external fan. And the velocity is set as $15.0 \mathrm{~m} / \mathrm{s}$. The outlet boundary condition is outflow.

2) The inlet velocity is chosen as the inlet boundary condition of the external ventilated path of the cooler. And the velocity is set as $5.0 \mathrm{~m} / \mathrm{s}$. Furthermore, the temperature of the cryogenic fluid is $23{ }^{\circ} \mathrm{C}$, defining the outlet of the external ventilated path as pressure-outlet.

3) The inlet velocity is chosen as the inlet boundary condition of the internal ventilated path of the cooler. And the velocity is set as $6.5 \mathrm{~m} / \mathrm{s}$. Further, the temperature of the high-temperature fluid is $75{ }^{\circ} \mathrm{C}$, defining the outlet of the internal ventilated as pressure-outlet.

\subsection{Experimental rig}

In order to verify the accuracy of the results of the modelling simulation, an experimental rig of the external ventilated path of the motor is setup in this research. The experimental rig including the prototype, the thermistors and the sensors is shown in Figure 6. Under the condition of safe and stable operation of the prototype, multi point measurement is adopted to improve the accuracy of the test data.

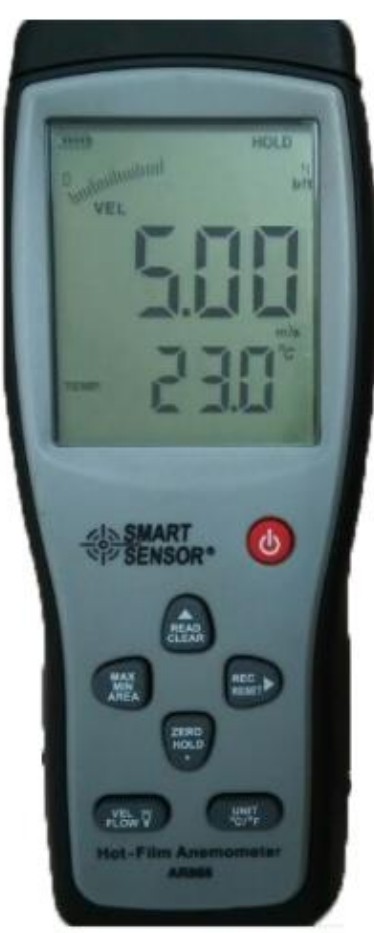

(1) Sensor

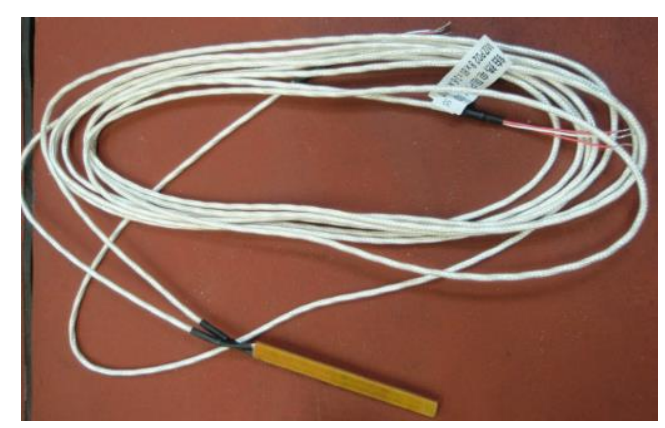

(2) Thermistor

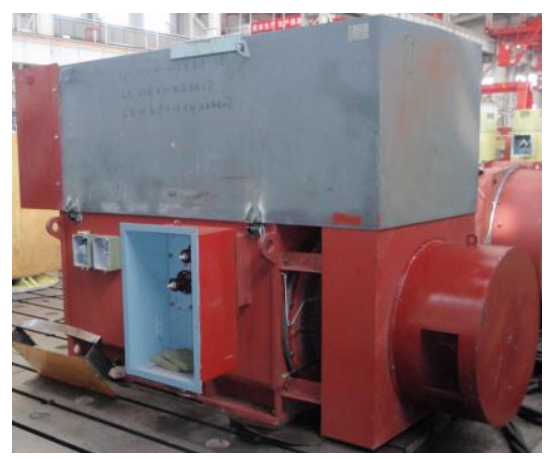

(3) Prototype

Fig.6. The experimental rig 


\section{Results and discussion}

\subsection{The external fan}

The external fan is the core part of the external ventilated path and directly affects the heat dissipation of the motor. So it is important to study external fan performance. The external fan area can be divided into two parts: static region (front and back plate) and the rotation region (blades of external fan). For the rotation region, the multiple reference frame (MRF) method is used to analyze. The external fan area is modelled and simulated by the software ANSYS Fluent.

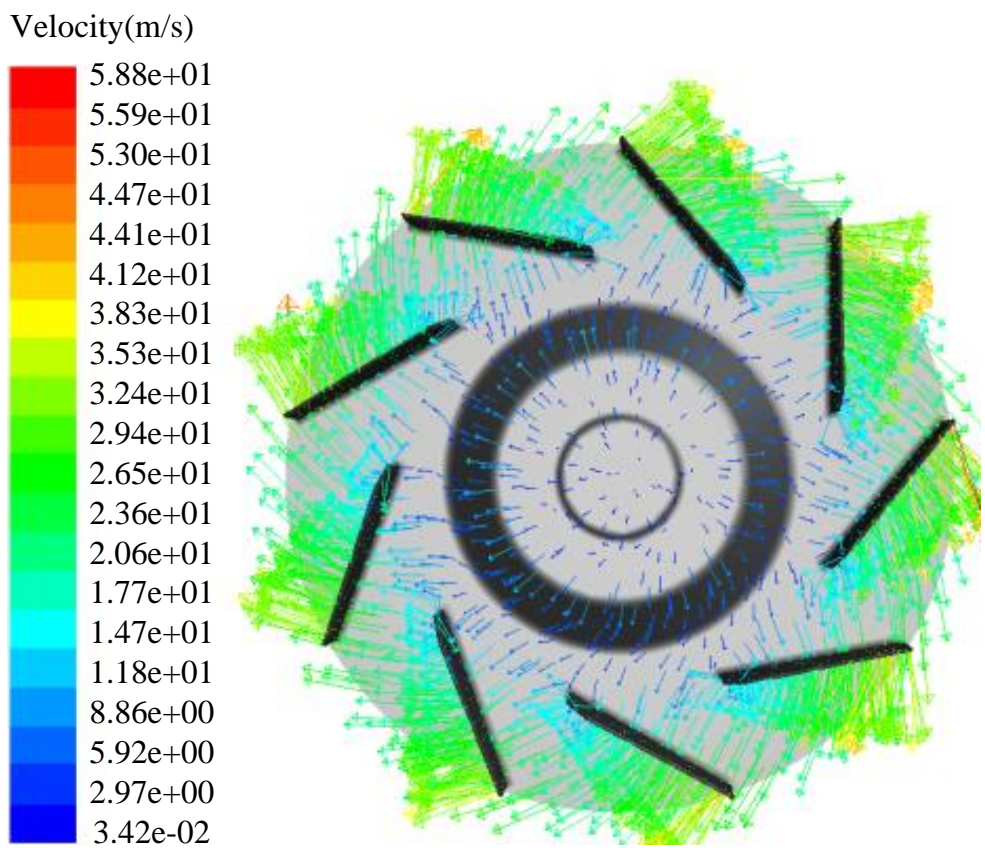

Fig.7.Blade surface fluid velocity vector

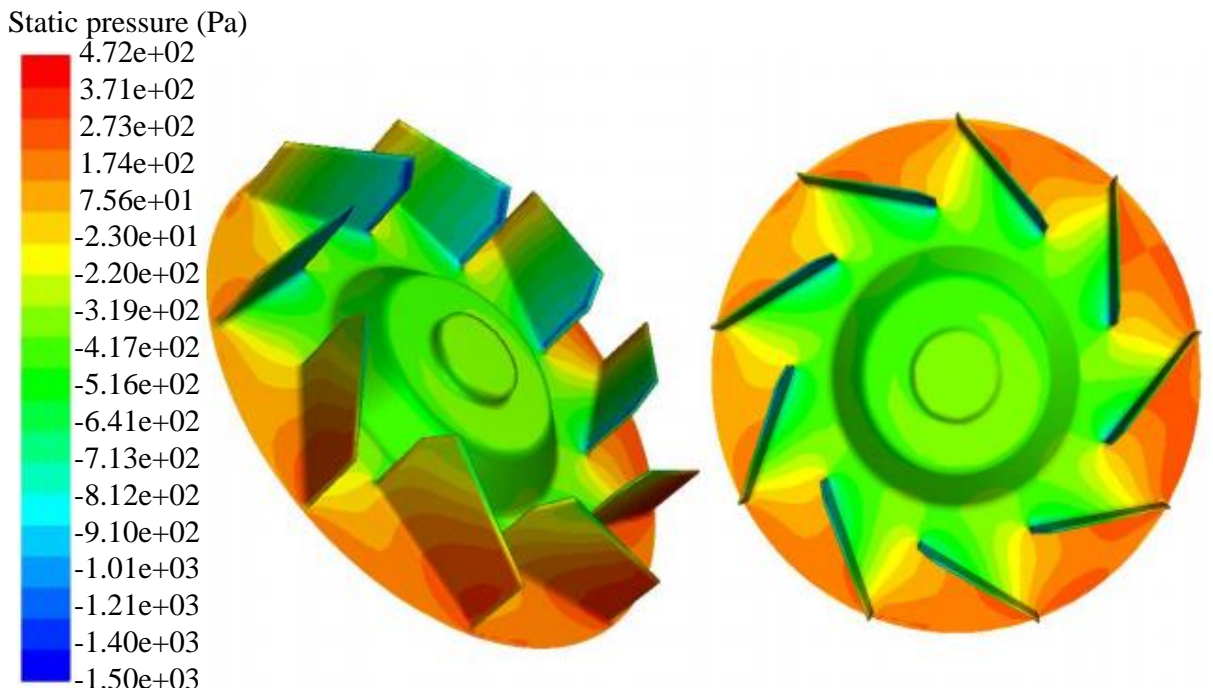

Fig.8. Static pressure cloud picture of fan

The velocity vector diagram and static pressure cloud-picture of the external fan 
rotation area are obtained by numerical calculation as shown in Fig.7 and Fig.8 respectively. In Fig.7, the windward side of the fan blades is smaller than the leeward side. The movement direction of the fluid on the blade surface is rotated clockwise in the center of the fan axis. The nearer to the edge of the blades, the greater the velocity, the maximum value is $6 \mathrm{~m} / \mathrm{s}$. It also shows that the outer edge has more heat than the inner. This is also illustrated in the pressure cloud-picture in Figure 8. The pressure on the leeward side of the blades is greater than the windward side. The greater the pressure is when it is nearer to the outer edge of the external fan. By contrast, the windward side bears more negative pressure, and the nearer to the circle direction, the lower pressure. This is because the air in front of the fan blades is pushed forward and the air flow velocity is greater than that of the leeward side of the fan blades when the fan blades rotate. the pressure of the local pressure is small, so the pressure of the leeward side of the fan blades is larger than that of the fan windward.

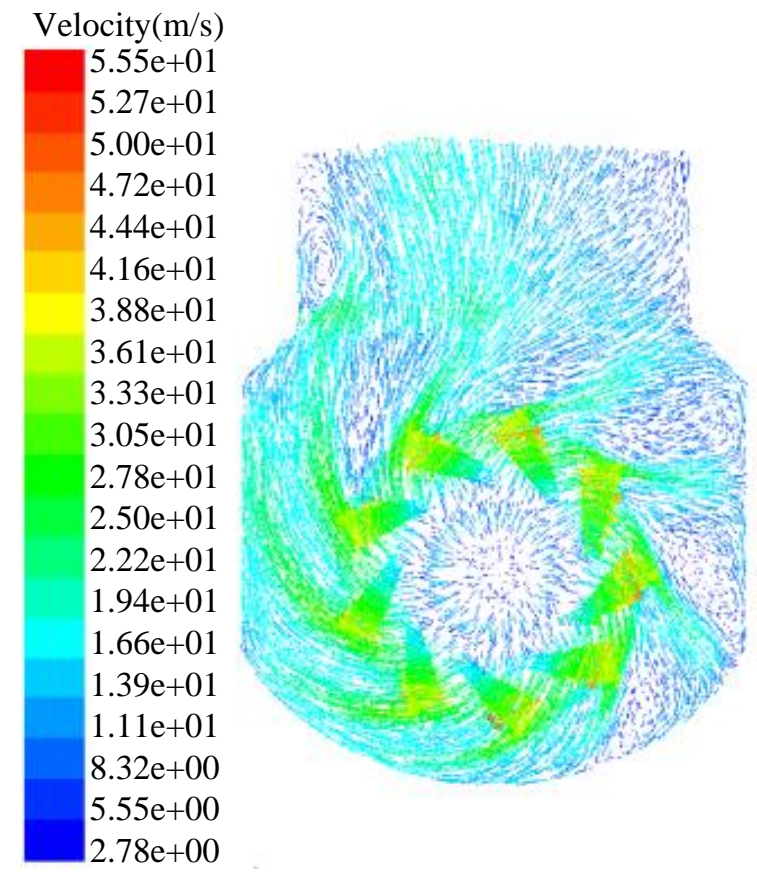

Fig.9. The fluid velocity on central section

Fig.9 shows the fluid velocity on the central section. The figure shows that the fluid velocity on left side is greater than the right. This is caused by the external fan rotation. It also indicates that the fluid velocity increases from the blades along the direction of rotation. Because the left velocity is greater than the right, it is inferred that the velocity of the right side is less than the left side, but not uniformed, when the fluid enters the cooler. This is consistent with the results from the experimental tests.

\subsection{Optimal design of the external fan}

There are three main parameters which can influence on the fan performance: number of fan blades, deflection angle and outlet angle of fan blade. Fan blade structure is shown in Fig.10. The relationship between the number of blades and the fan efficiency can be found in paper [25]. The fan efficiency can be improved with the 
increase of the number of blades, but it will reaches to the maximum and no longer changes when increased to a certain number. Because of the reason, the optimal design of the external fan in this study is only concentrated on changing the deflection angle and outlet angle of fan blades. The deflection angle of fan blades is changed firstly to get high fan efficiency from various angles. Then the outlet velocity of fan is obtained by optimizing the outlet angle of fan blades with the maximum efficiency at the certain deflection angle. The results of comparison optimization of fan efficiency and outlet velocity is shown in Fig. 11.

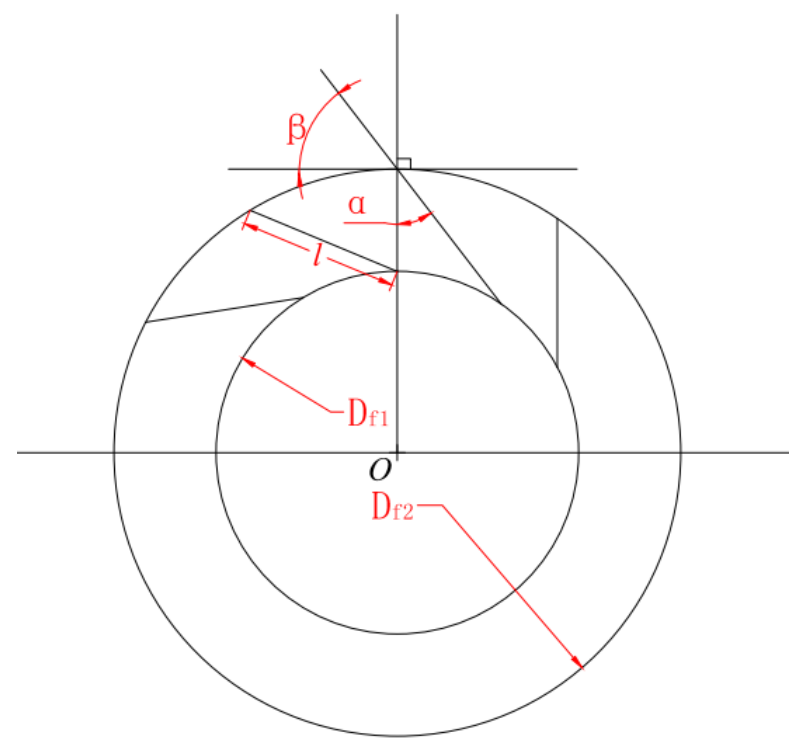

$D_{f 1}$ and $D_{f 2}-$ inner and outer diameter of the fan; $l$-the length of fan blades; $\alpha=40^{\circ}-$ deflection angle fan blades, $\beta=50^{\circ}$ - outlet angle of the fan.

Fig.10.The structure of fan blades

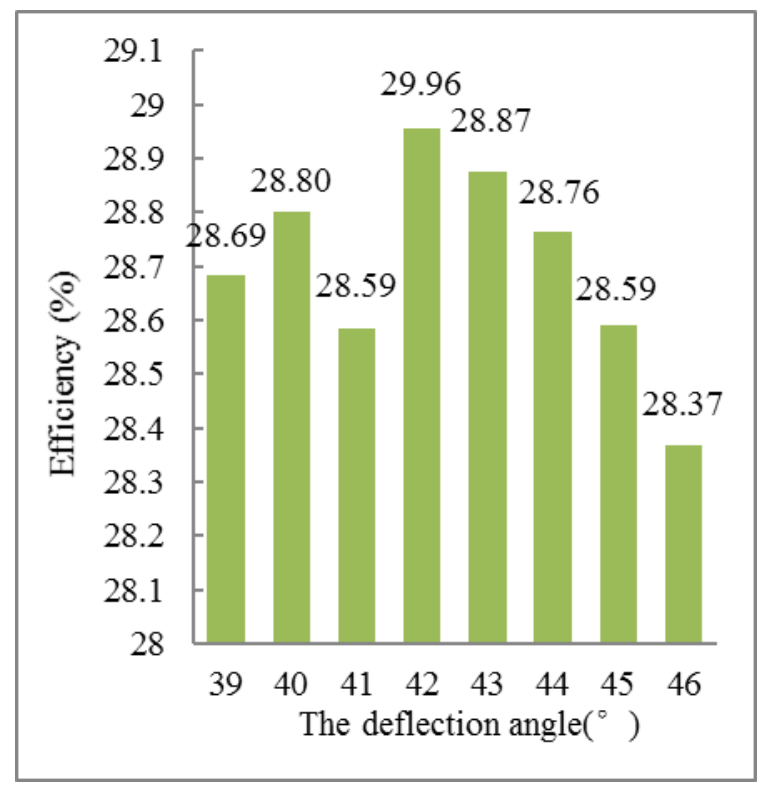

(a) Comparison of efficiency by different deflection angle of fan blades 


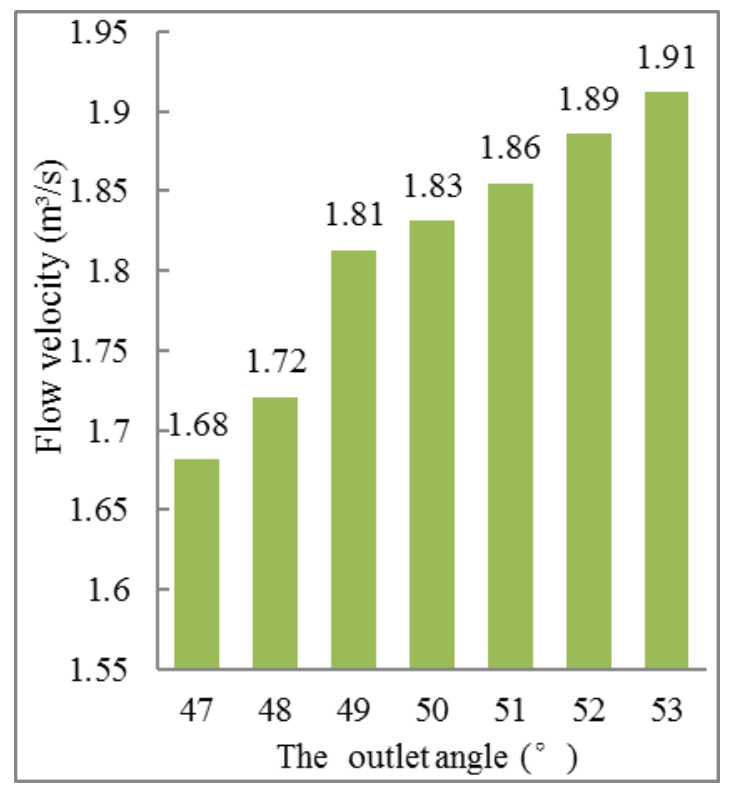

(b) Comparison of flow velocity by different outlet angle of fan

Fig.11. Comparison optimization 
Figure 11 (a) shows the effect of fan blade deflection angle on efficiency. From the figure, it can be seen the maximum efficiency of fan is $29.96 \%$, when $\alpha=42^{\circ}$. There is a close relationship between the outlet flow and the outlet angle of the fan blades, as shown in Figure 11(b). When $\beta=53^{\circ}$, the outlet flow is the largest which is $1.91 \mathrm{~m}^{3} / \mathrm{s}$ and is increased by $0.08 \mathrm{~m}^{3} / \mathrm{s}$. Fan performance is greatly improved by selecting $\alpha=42^{\circ}, \beta=53^{\circ}$. Improving the performance of the fan also greatly improve the cooling and the safety of the motor in operation. As a result, it can improve the capacity of the motor.

\subsection{Heat transfer characteristics of cooler}

In the above section, the performance of the external fan is studied and analyzed. The outlet velocity of air flow from the external fan is used as the inlet velocity of the cooler. A 3D model of cooler, as shown in Fig. 5, is modelled in Fluent.

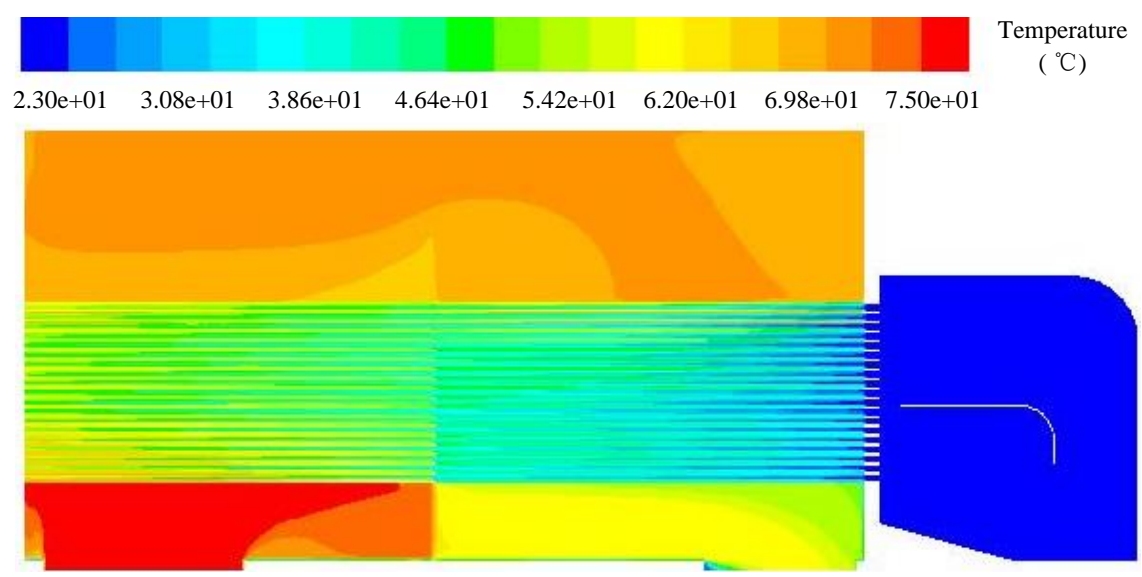

Fig.12.The cloud picture of temperature distribution in the cooler

Fig.12 is the cloud picture of temperature distribution from the Fluent modelling. From Fig.12, it can be seen the temperature of hyperthermal fluid of internal ventilated path is reduced from $75^{\circ} \mathrm{C}$ to $53.7^{\circ} \mathrm{C}$ by transferring the heat with the cooling pipe wall. And the temperature of cryogenic fluid of external ventilated path is rose from $23^{\circ} \mathrm{C}$ to $49.4^{\circ} \mathrm{C}$. It is consistent with the law of conservation of energy. The distribution of cooler temperature on the left side is higher than the right, because the left side is directly connected with the hyperthermal fluid of the internal ventilated path. Because of the uneven temperature distribution, the cooling effect has not reached to the best. 


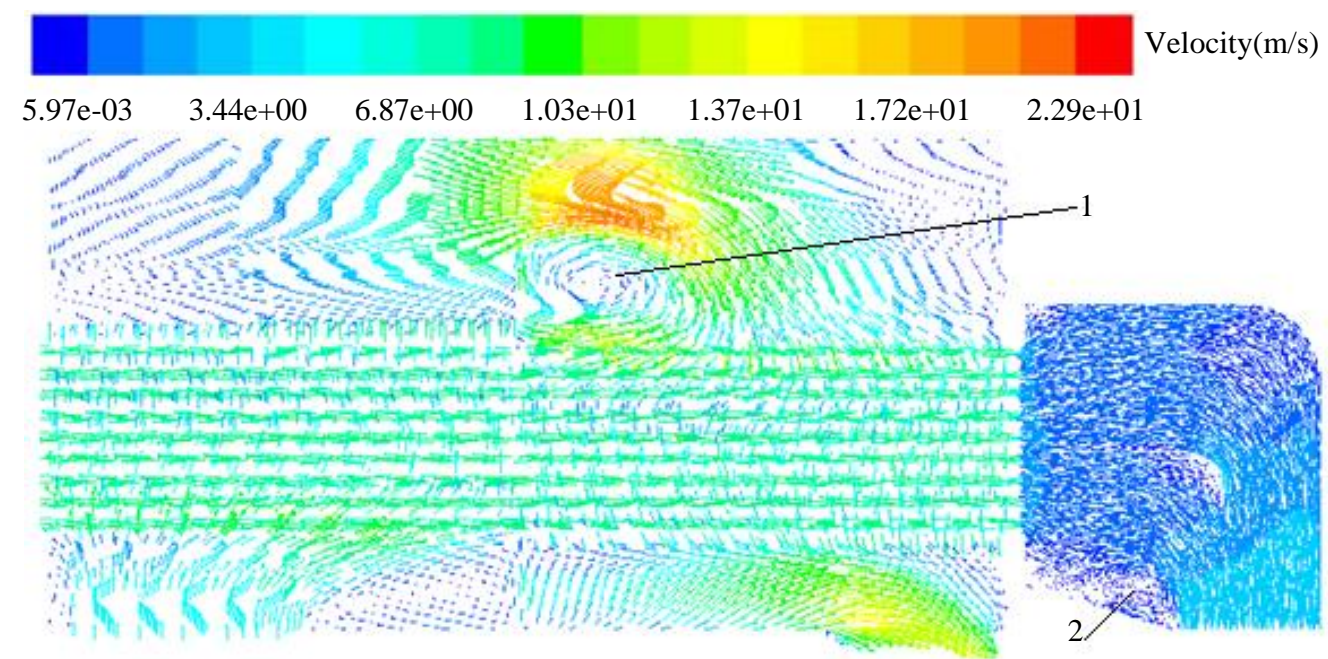

Fig.13.The velocity vector of cooler

Fig.13 shows the velocity vector of the cooler. From the figure, it can be seen the velocity of the fluid at the top of the windshield is increased greatly after flowing through the windshield because this cross section of the fluid flow is decreased. The air velocity at the outlet of internal ventilated path illustrate this effect. The heat transfer capacity of the corresponding high velocity is strong. There are obvious vortexes at location 1 and 2 in Fig. 13. The phenomenon of vortex causes the energy loss and reduces the efficiency of the motor ventilated system. Therefore it is necessary to avoid them as much as possible.
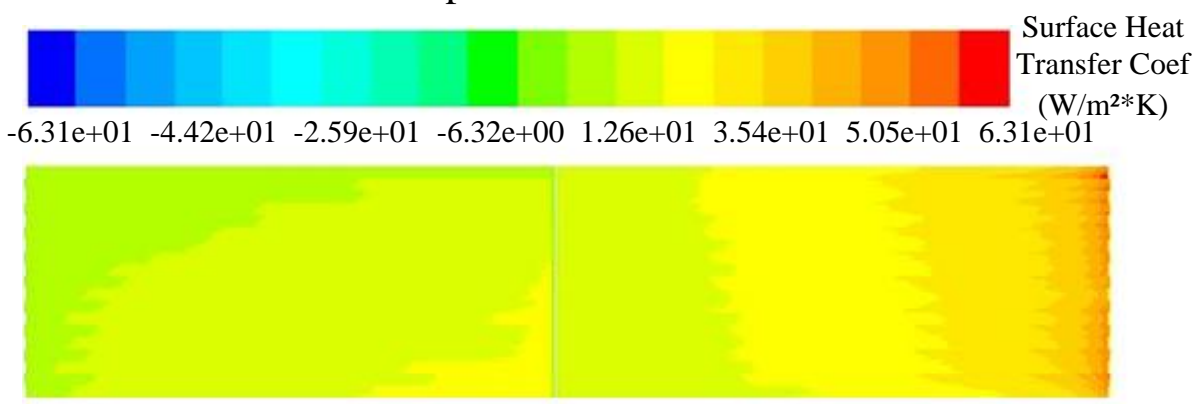

a) Convective heat transfer coefficient of aluminum tube inner surface

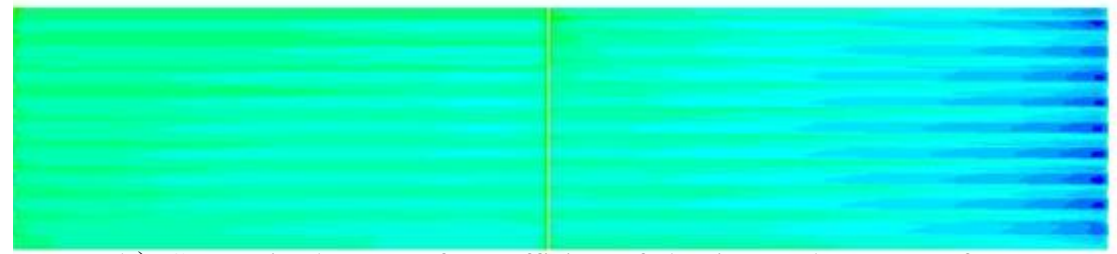

b) Convective heat transfer coefficient of aluminum tube outer surface

Fig. 14. Convective heat transfer coefficient of aluminum tube surface

Fig. 14 presents the convective heat transfer coefficient of aluminum tube surface. The figure shows that the heat transfer capacity of the cryogenic fluid in the external ventilated path is significantly increased when it enters the cooling aluminum pipe. When the cryogenic fluid flows into the cooling pipe, the cross section of fluid decreases suddenly and makes the fluid velocity a sharp increase, which results in the 
improvement of heat dissipation capacity. From Fig.14 (a), it can be found that the heat transfer coefficient on the right side is significantly higher than the left. The nearer to outlet of aluminum pipe, the smaller convective heat transfer coefficient is. In Fig. 14 (b), the heat transfer coefficient of right side is also obviously higher than the left. The positive and negative of heat transfer coefficients represent different directions. The inner surface of the cooling aluminum pipe transmits heat to the inside of the cooler, so the convective heat transfer coefficient is positive. Conversely, the outer surface of the aluminum pipe absorbs heat, so the convective heat transfer coefficient is negative.

\subsection{Optimal design of the cooler}

As mentioned above, an obvious vortex is found at location 1 in Fig. 13. In order to solve the problem, the height of windshield is changed to reduce the vortex and optimize the physical performance of the cooler. It is found the vortex can be reduced by lowering the height of the windshield. The reduction of vortex can reduce energy loss and noise, meanwhile improve the efficiency of the motor. The windshield height does not affect the outlet temperature of the internal ventilated path, but it has a great influence on inlet static pressure of the internal ventilated path. The comparison of static pressure is shown in Fig. 15. From the figure, it can be seen the minimum static pressure appears when the windshield height is reduced by $132 \mathrm{~mm}$.

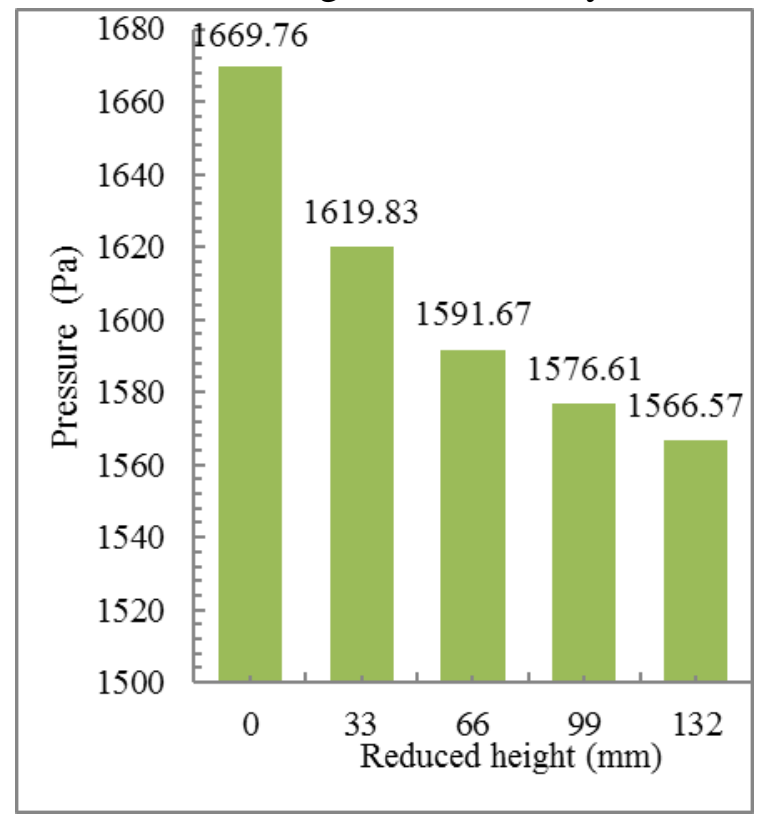

Fig. 15. Contrast of static pressure

It is known that the cooling capacity of the cooler can be improved by increasing the number of the windshield. Fig. 16 shows the contrast of the outlet temperature to the internal ventilated path. From Figure 16, it can be found that the outlet temperature of the internal ventilated path dropped by $2.3{ }^{\circ} \mathrm{C}$ when the number of windshield increased from 1 to 3 . The result shows that the heat transfer performance of the internal and external ventilation paths is improved. The result can provide a basic method for further improving the power density of motor. 


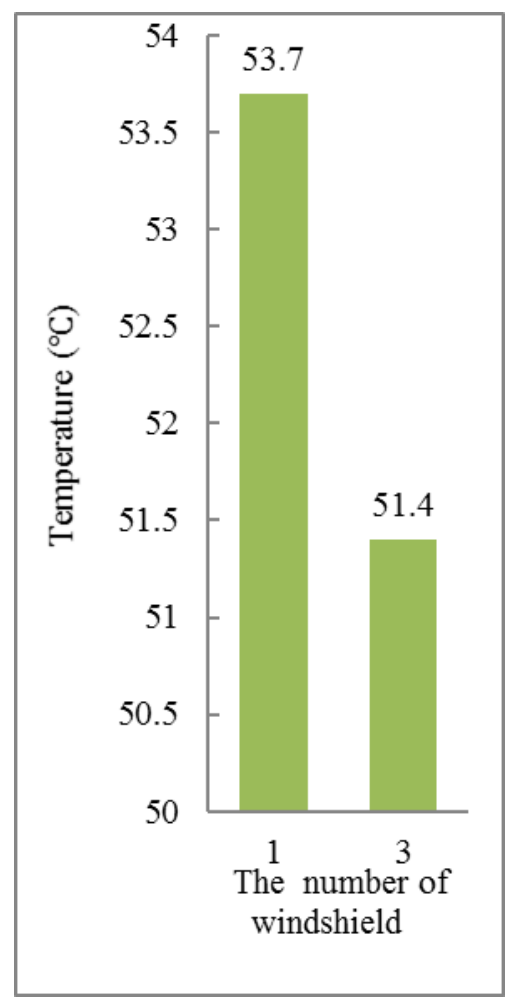

Fig. 16. Contrast of the outlet temperature in the internal ventilated path

The Inclined plate near vortex at position 2 in Fig.13 is replaced by arc plate. Velocity vector of cooler is re-modelled and re-simulated. Fig. 17 presents the contrast of velocity vector with different shapes of inclined plate. Fig.17 shows the vortex is reduced and energy loss and noise are also reduced. Improvement of the cooler performance can also enhance the safety of the motor operation greatly.

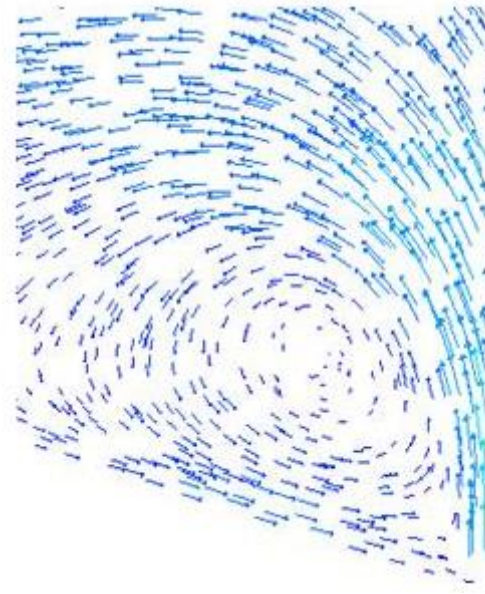

(a) Before optimization

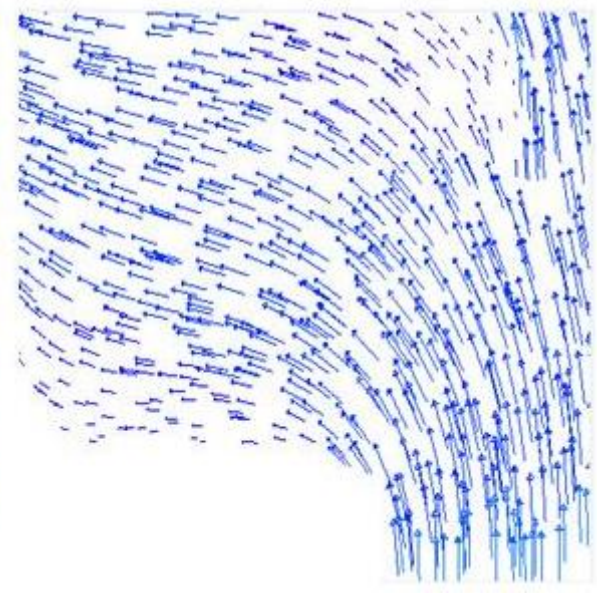

(b) Postoptimality

Fig.17. Contrast of Velocity vector with different shape of inclined plate

Fig. 18, 19 and 20 shows the cloud picture of temperature distribution after postoptimality, the velocity vector diagram of postoptimality and the convective heat transfer coefficients of postoptimality respectively. 


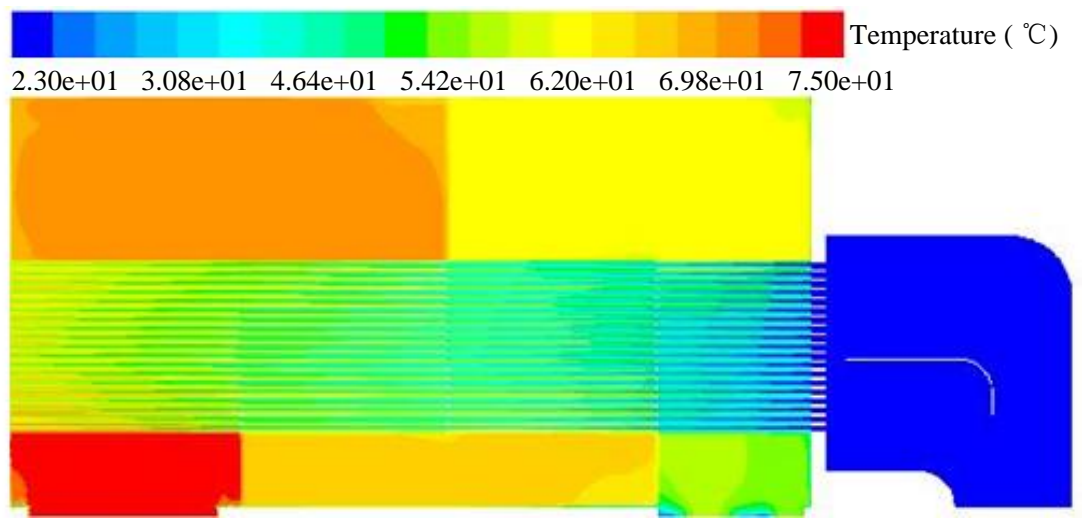

Fig.18. The temperature cloud picture of postoptimality on profile section of cooler

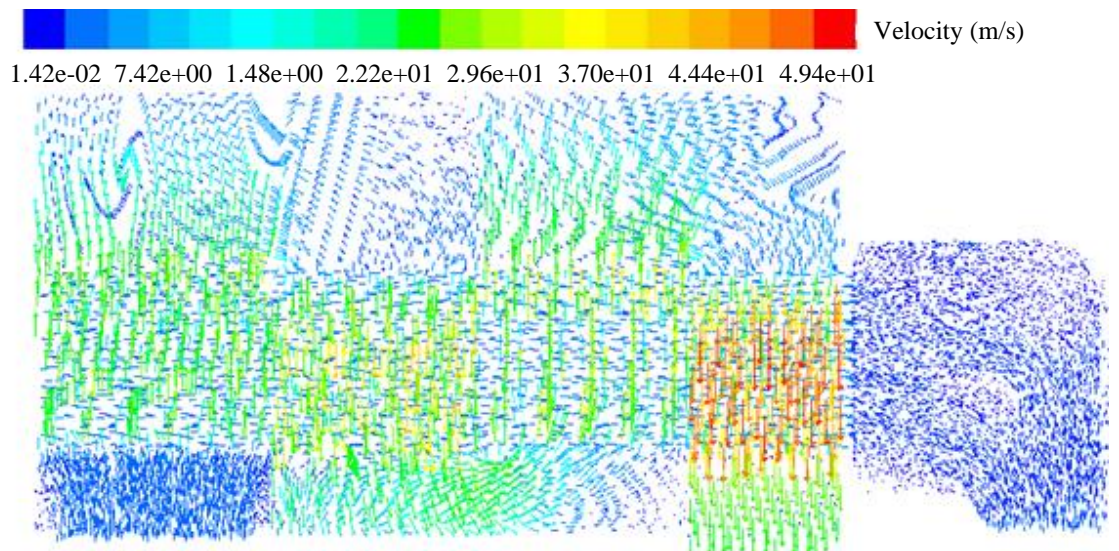

Fig.19.The velocity vector diagram of postoptimality on profile section of cooler

Fig.18 presents the temperature distribution of the cooler after postoptimality. From the figure, it can be seen the outlet temperature of internal fluid of the post-optimized cooler is $50.7^{\circ} \mathrm{C}$, and the outlet temperature of external fluid is $51.9{ }^{\circ} \mathrm{C}$. After changing the outlet temperature, the internal fluid temperature is dropped by $3{ }^{\circ} \mathrm{C}$ and the external fluid temperature is increased by $2.5^{\circ} \mathrm{C}$, the cooling capacity of the cooler is significantly improved. It shows that the heat transfer capability of internal and external ventilated path is greatly enhanced and the ability of secure and stable operation of the motor is significantly strengthened.

Fig.19 shows that the vortex phenomenon of cooler after postoptimality is reduced and the flow velocity of the post-optimized cooling pipe is greater than that of the before-optimized. The reason is that energy loss is reduced with the decrease of the vortex in the air duct meanwhile the flow velocity is increased. The increase of flow velocity can make the cold air take away more heat in the same time the heat transfer coefficient is improved.

Fig.20 shows the convective heat transfer coefficients in aluminum surface after postoptimality. The convective heat transfer coefficient is higher than that before the model changes which reduces the temperature of the internal ventilation path. 


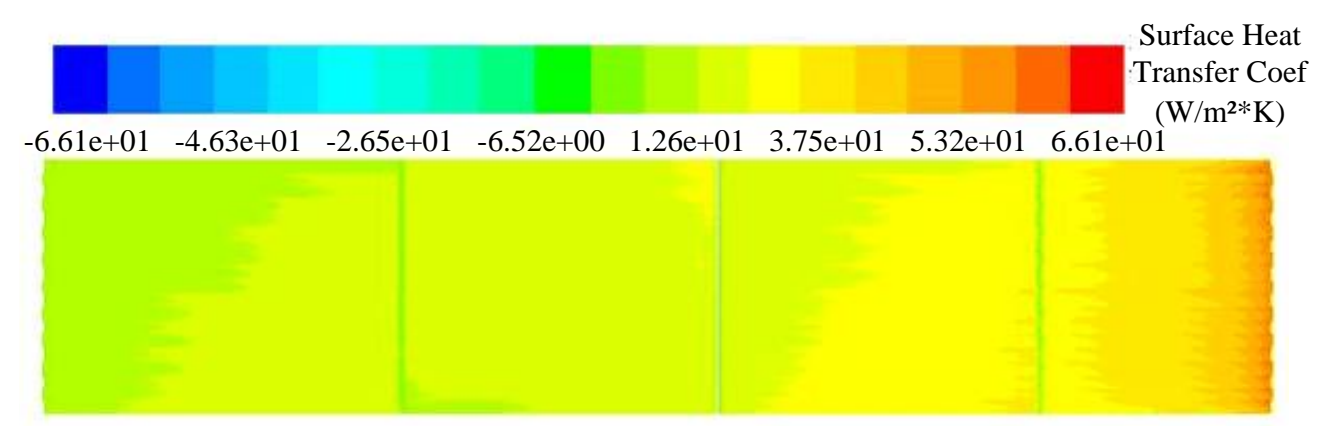

a) Convective heat transfer coefficient of aluminum tube inner surface

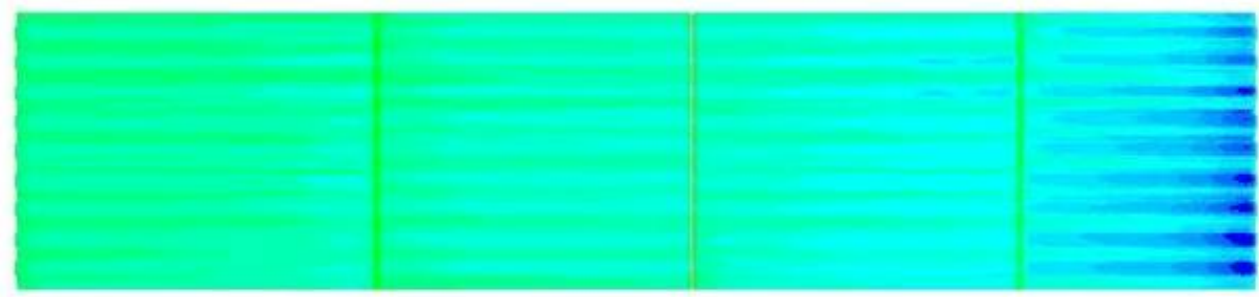

b) Convective heat transfer coefficient of aluminum tube outer surface

Fig.20. The convective heat transfer coefficients in aluminum surface of postoptimality

\section{Experimental results}

As described in 2.4, an experimental rig of the external ventilated path of the motor is setup. A series of tests on the outlet and inlet velocity and outlet temperature of internal and ventilated path of the compact high-voltage motor prototype are performed. The test results are shown in Table 1. During the testing, sensors are placed at multiple measurement points for multiple measurements, and then the average values are taken. Table 1 shows the contrast of the results from experimental tests and computational simulations.

Table 1 Contrast of experimental data and theoretical calculation

\begin{tabular}{c|c|c|c}
\hline & Theoretical calculation & Experimental data & Relative error \\
\hline $\begin{array}{c}\text { Outlet velocity of the } \\
\text { external fan( } \mathrm{m} / \mathrm{s})\end{array}$ & 5.15 & 5.0 & $3.0 \%$ \\
\hline $\begin{array}{c}\text { Inlet velocity of the } \\
\text { internal ventilated path } \\
(\mathrm{m} / \mathrm{s})\end{array}$ & 6.5 (given) & 6.5 & - \\
\hline $\begin{array}{c}\text { Inlet velocity of the } \\
\text { external ventilated path } \\
(\mathrm{m} / \mathrm{s})\end{array}$ & 5.0 (given) & 5.0 & $-3.5 \%$ \\
\hline $\begin{array}{c}\text { Outlet velocity of the } \\
\text { internal ventilated path } \\
(\mathrm{m} / \mathrm{s})\end{array}$ & 11.77 & 11.37 & $3.8 \%$ \\
\hline $\begin{array}{c}\text { Outlet velocity of the } \\
\text { external ventilated path } \\
(\mathrm{m} / \mathrm{s})\end{array}$ & 7.72 & 7.44 & $3.7 \%$ \\
\hline Outlet temperature of the & 53.7 & 55.7 & \\
\hline
\end{tabular}




\begin{tabular}{c|c|c|c}
\hline $\begin{array}{c}\text { internal ventilated path } \\
\left({ }^{\circ} \mathrm{C}\right)\end{array}$ & & \\
\hline $\begin{array}{c}\text { Outlet temperature of the } \\
\text { external ventilated path } \\
\left({ }^{\circ} \mathrm{C}\right)\end{array}$ & 49.4 & 51.6 & $4.8 \%$ \\
\hline
\end{tabular}

From the table, it can be seen the experimental value of outlet velocity of the internal ventilated path and external ventilated path are $11.37 \mathrm{~m} / \mathrm{s}$ and $7.44 \mathrm{~m} / \mathrm{s}$ respectively, compared to the results from simulation $11.77 \mathrm{~m} / \mathrm{s}$ and $7.72 \mathrm{~m} / \mathrm{s}$. Both are small than the theoretical calculation values. However, the relative error between them is less than 4\%. Experimental value of outlet temperature of the internal ventilated path and external ventilated path respectively are $55.7^{\circ} \mathrm{C}$ and $51.6^{\circ} \mathrm{C}$. Both are larger than the theoretical calculation values. Nevertheless, the relative error between them is less than $5 \%$.

From the table, it can be seen the relative error between experimental and simulation results are within the range of the allowable error of the engineering application. It is proved that the method of modeling and simulation is reliable.

\section{Conclusions}

The heat transfer characteristics of External Ventilated Path of a compact, high-voltage, 4-pole, $2500 \mathrm{~kW}$ motor is studied by flow-thermal coordination mechanism. From the modelling and simulation, the characteristics of fluid distribution of the external fan is found. The flow velocity of the fluid on the left side of the external fan is greater than that of the right. The characteristics of the fluid flow and temperature distribution of the cooler are obtained.

The performance of external fan is improved by changing the deflection angle ( $\alpha=42^{\circ}$ ) and outlet angle $\left(\beta=53^{\circ}\right.$ ) of fan blades. The efficiency of external fan is improved from $28.80 \%$ to $29.96 \%$ and outlet flow was increased by $0.08 \mathrm{~m}^{3} / \mathrm{s}$. Some optimal measures are proposed to improve the performance of the cooler such as adjusting the height of the windshield, increasing the number of the windshield, changing the shape of inclined plate. The temperature of hyperthermal fluid of inner ventilated path is decreased from $75^{\circ} \mathrm{C}$ to $53.7^{\circ} \mathrm{C}$ by transferring heat to the cooling pipe wall, at the same time the temperature of cryogenic fluid of external ventilated path is increased from $23^{\circ} \mathrm{C}$ to $49.4^{\circ} \mathrm{C}$. The outlet temperature of internal fluid of post-optimized cooler is dropped by $3^{\circ} \mathrm{C}$, and the external fluid temperature is increased by $2.5{ }^{\circ} \mathrm{C}$. The cooling capacity of the cooler is improved, and it is beneficial to the safe and stable operation of the motor.

The outlet velocity and temperature of the internal ventilated path and external ventilated path are verified using experimental results. The accuracy of method of modeling and simulation is proved. The results from the study can provide an effective tool for the design of high power density induction motors. 


\section{Acknowledgements}

This research is supported by the project (51207036) of National Natural Science Foundation of China.

\section{References:}

1. Naskar, A.K. and D. Sarkar. Numerical analysis of three dimensional steady state heat conduction in the rotor of an induction motor by finite element method. in International Conference on Control, Instrumentation, Energy and Communication. 2014.

2. Ding, S., et al., Numerical research of wind friction loss for doublefed hydro-turbine generator. Journal of Huazhong University of Science \& Technology, 2014.

3. Chang, C.C., et al., Air cooling for a large-scale motor. Applied Thermal Engineering, 2010. 30(11-12): p. 1360-1368.

4. Li H. Cooling of a permanent magnet electric motor with a centrifugal impeller[J]. International Journal of Heat \& Mass Transfer, 2010, 53(4):797-810.

5. Li H. Flow driven by a stamped metal cooling fan - Numerical model and validation[J]. Experimental Thermal \& Fluid Science, 2009, 33(4):683-694.

6. Stafford J, Walsh E, Egan V. Local heat transfer performance and exit flow characteristics of a miniature axial fan[J]. International Journal of Heat \& Fluid Flow, 2010, 31(5):952-960.

7. Ding S, Liu J, Zhang L. Fan characteristics of the self-support components of rotor ends and its performance matching $\mathcal{\zeta}[\mathrm{J}]$. International Journal of Heat \& Mass Transfer, 2017, 108:1917-1923.

8. Xie, Y. and Y. Wang, 3D temperature field analysis of the induction motors with broken bar fault. Applied Thermal Engineering, 2014. 66(1-2): p. 25-34.

9. Herbert, W.A., Totally Enclosed Fan-Cooled Squirrel-Cage Induction Motor Options. IEEE Transactions on Industry Applications, 2014. 50(2): p. 1590-1598.

10. Ahmed, F., E. Ghosh, and N.C. Kar. Transient thermal analysis of a copper rotor induction motor using a lumped parameter temperature network model. in IEEE Transportation Electrification Conference and Expo. 2016.

11. Zhang, Y., et al., Calculation of Temperature Rise in Air-cooled Induction Motors Through 3-D Coupled Electromagnetic Fluid-Dynamical and Thermal Finite-Element Analysis. Magnetics IEEE Transactions on, 2012. 48(2): p. 1047-1050.

12. Kolondzovski, Z., A. Belahcen, and A. Arkkio, Multiphysics thermal design of a high-speed permanent-magnet machine. Applied Thermal Engineering, 2009. 29(13): p. 2693-2700.

13. Kim C, Lee K S, Kim C, et al. Numerical investigation of the air-gap flow heating phenomena in large-capacity induction motors[J]. International Journal of Heat \& Mass Transfer, 2017, 110:746-752.

14. Nategh, S., et al., Thermal Modeling of Directly Cooled Electric Machines Using Lumped Parameter and Limited CFD Analysis. IEEE Transactions on Energy Conversion, 2013. 28(4): p. 979-990.

15. Torriano, F., et al., Heat transfer coefficient distribution on the pole face of a hydrogenerator scale model. Applied Thermal Engineering, 2014. 10(1): p. 153-162. 
16. Nair, D.G., T. Jokinen, and A. Arkkio. Coupled analytical and 3D numerical thermal analysis of a TEFC induction motor. in International Conference on Electrical Machines and Systems. 2015.

17. Xu, C., Application of Heat-Pipe Heat Exchanger to Electromotor Cooler. Basic Automation, 1999.

18. Wen, J. and J. Zheng, Numerical analysis of the external wind path for medium-size high-voltage asynchronous motors. Applied Thermal Engineering, 2015. 90: p. 869-878.

19 Wang Fujun, Computational Fluid Dynamics Analysis e CFD Principle and Application, Tsinghua University Press, Beijing, 2004, pp. 7-12.

20. Wardsmith, A.J., Internal fluid flow - The fluid dynamics of flow in pipes and ducts. Nasa Sti/recon Technical Report A, 1980. 81.

21. Chen, Q.G., X.U. Zhong, and Y.J. Zhang, Application of RNG k-?Models in Numerical Simulations of Engineering Turbulent Flows. Chinese Quarterly of Mechanics, 2003.

22. Fujita, M., et al. Air-cooled large turbine generator with multiple-pitched ventilation ducts. in IEEE International Conference on Electric Machines and Drives. 2005.

23. Li, W., J. Cao, and X. Zhang, Electrothermal Analysis of Induction Motor With Compound Cage Rotor Used for PHEV. Industrial Electronics IEEE Transactions on, 2010. 57(2): p. 660-668.

24. Rajagopal, M.S., K.N. Seetharamu, and P.A. Ashwathnarayana, Transient thermal analysis of induction motors. IEEE Transactions on Energy Conversion, 1998. 13(1): p. 62-69.

25. Shimbara N, Kodama Y, Hayashi H, et al. Characteristics of Fluid Dynamics and Noise of a Multiblade Radial Fan. Effects of inner diameter and the number of blades on the characteristics of fluid dynamics and noise.[J]. Nihon Kikai Gakkai Ronbunshu B Hen/transactions of the Japan Society of Mechanical Engineers Part B, 1996, 62(602):3642-3648. 